\title{
Infants' responsiveness to the auditory and visual attributes of a sounding/moving stimulus
}

\author{
DAVID J. LEWKOWICZ \\ New York State Institute for Basic Research in Developmental Disabilities, Staten Island, New York
}

\begin{abstract}
Responses to unimodal and multimodal attributes of a compound auditory/visual stimulus were investigated in 4-, 6-, 8-, and 10-month-old infants. First, infants were habituated to a compound stimulus consisting of a visual stimulus that moved up and down on a video monitor and a sound that occurred each time the visual stimulus reversed direction at the bottom. Once each infant met a habituation criterion, a series of test trials was administered to assess responsiveness to the components of the compound stimulus. Response was defined as the total duration of visual fixation in each trial. In the two unimodal test trials, the rate at which the component was presented was changed while the rate of the other component remained the same, whereas in the bimodal test trial the rate of both components was changed simultaneously. Results indicated that infants at each age successfully discriminated the bimodal and the two unimodal changes and that regression to the mean did not account for the results. Results also showed that disruption of the temporal relationship that accompanied the change in rate in the two unimodal test trials was also discriminable, but rate changes appeared to play a greater role in responsiveness than did synchrony changes. Considered together with results from similar prior studies, the current results are consistent with the modality appropriateness hypothesis in showing that discrimination of temporal changes in the auditory and visual modalities is dependent on the specialization of the sensory modalities.
\end{abstract}

Many objects and events in the infant's world are specified by concurrent auditory and visual attributes. Many of these attributes (e.g., duration, rhythm, or intensity) are equivalent across modalities, and, in order for the infant to perceive objects and events as perceptually unified, he/she must be able to perceive the intersensory equivalence specified by these attributes. Adults are known to possess the ability to unify heteromodal inputs on the basis of equivalent attributes (Marks, 1978; Welch \& Warren, 1986). Infants also appear to possess some of these abilities. For example, it has been reported that infants can detect auditory-visual equivalence on the basis of intensity (Lewkowicz \& Turkewitz, 1980), rate (Spelke, 1979), rhythm (Allen, Walker, Symonds, \& Marcell, 1977; Mendelson \& Ferland, 1982), linguistic content (Kuhl \& Meltzoff, 1982), and synchrony (Bahrick, 1987; Lewkowicz, in press) as early as 3-4 months of age, and on the basis of duration/synchrony by 6 months of age (Lewkowicz, 1986).

Although the studies on infants' ability to detect intersensory equivalence provide important information about

\footnotetext{
This work was supported in part by funds from the New York State Office of Mental Retardation and Developmental Disabilities and NICHD Grant HD 24371. I thank Marci Dabbene for her assistance in data collection and Judith Gardner, Gilbert Gottlieb, and Gerald Turkewitz for helpful comments on an earlier version of the manuscript. Correspondence should be addressed to D. J. Lewkowicz, N.Y.S. Institute for Basic Research in Developmental Disabilities, 1050 Forest Hill Rd., Staten Island, NY 10314 (e-mail: ddlsi@cunyvm).
}

the development of intersensory integration mechanisms, they address only one aspect of intersensory integration. Under normal circumstances, when infants are faced with multimodally specified objects or events, they can respond either to the intersensory equivalence specified by the auditory and visual attributes or just to the input in one of the modalities, ignoring the input in the other modality. The design of the studies of infants' response to intersensory equivalence does not permit an assessment of the latter possibility. To determine if infants might resort to a single modality response strategy, they must be presented with auditory/visual compounds where a response to each component can be assessed independently.

The first systematic examination of infants' response to auditory/visual compounds was carried out by Lewkowicz (1988a, 1988b). In these studies, 6- and 10-month-old infants first were habituated to a compound stimulus consisting of a checkerboard that flashed periodically and a tone that was sounded periodically. Once they became habituated to the compound stimulus, the infants were given a series of test trials to determine which aspect of the compound stimulus they attended to during habituation. This was done by changing the temporal characteristics (i.e., rate and/or duration) of either the visual or the auditory component while keeping the temporal characteristics of the other component unchanged, or by changing the temporal characteristics of both components simultaneously. The dependent measure was the total duration of visual fixation in each trial, and the measure of discrimination was recovery of visual fixation following habituation. 
Results indicated that both age groups discriminated the simultaneous change in both components, but that they differed in their response to the two unimodal changes. The 6-month-old infants discriminated the changes in the auditory component but not the visual component, regardless of whether rate alone, or rate and duration, were changed, and regardless of whether the temporal relationship of the auditory and visual components was concordant or discordant during habituation. In contrast, the 10month-old infants responded to changes in the visual component as well as to changes in the auditory component, although they still responded more to changes in the auditory component.

The failure of the 6-month-old infants to discriminate changes in the visual component was rather surprising given that the visual system is quite advanced by this age. Indeed, the failure to discriminate the visual changes occurred only when the visual component was presented in the presence of the auditory component. When the visual component was presented alone, changes in the rate of its presentation were discriminated by the 6 -month-old infants. The failure of the 6-month-old infants to discriminate the visual component changes, and the greater responsiveness of the 10-month-old infants to the auditory component changes, is consistent with the modality appropriateness hypothesis put forth by a number of workers investigating intersensory interactions in adults (Freides, 1974; Lederman, Thorne, \& Jones, 1986; O’Connor \& Hermelin, 1972; Welch \& Warren, 1986). The basic concept behind this hypothesis is that each modality is specialized for responding to specific stimulus features, and when inputs from different modalities are present at the same time, the perceiver will utilize and/or favor the modality best suited to the processing of the incoming information. In general, vision is best at processing spatial information, whereas audition is best at processing temporal information (Kubovy, 1988). The results from Lewkowicz's (1988a, 1988b) studies are consistent with the modality appropriateness hypothesis because the auditory component varied in temporal rate, whereas the temporal variations in the visual component were produced by a spatially static, and thus nonoptimal, visual stimulus.

The studies reported in the current article represent a further examination of infants' responsiveness to auditory/ visual compounds. According to the modality appropriateness hypothesis, infants' responsiveness to components of auditory/visual compounds should be affected by the degree to which the stimulation in each modality matches the specialization of the respective modalities. On the basis of the fact that infants respond more to moving displays than to spatially static ones (Volkmann \& Dobson, 1976; Wilcox \& Clayton, 1968) and that they respond differentially to different rates of visual motion early in infancy (Aslin \& Shea, 1990; Dannemiller \& Freedland, 1991; Freedland \& Dannemiller, 1987; Kaufmann, Stucki, \& Kaufmann-Hayoz, 1985; Lewkowicz, 1992), it seems reasonable to expect that infants would have little difficulty in detecting changes in the temporal characteristics of a visual component of an auditory/visual compound stim- ulus when the visual component is spatially dynamic. The purpose of the first experiment was to test this possibility.

\section{EXPERIMENT 1}

Each infant was habituated to a compound stimulus consisting of a visual stimulus that moved up and down on a video screen and a sound that occurred every time the stimulus reached the bottom of the screen. The overall impression created by this display was that of a bouncing object. As soon as the infant reached habituation criterion, one bimodal and two unimodal test trials were administered. In one unimodal test trial, the motion velocity (and thus rate) of the visual stimulus changed while the rate of the auditory component remained unchanged. In the second unimodal test trial, the repetition rate of the sound changed while the rate of the visual component remained unchanged. It should be noted that the change in the rate of the respective components in the two unimodal test trials, by definition, meant that there was also a change in the synchrony relation between the two components. The possible contribution of this second type of change will be addressed separately in Experiment 3. In the bimodal test trial, the rate of both components changed simultaneously. Recovery of response on a given test trial indicated whether the infant discriminated that particular change.

\section{Method}

Subjects. A total of 144 infants, consisting of separate groups of 36 infants at $4,6,8$, and 10 months of age, were tested. The mean age of the 4-month-old group was 19.9 weeks ( $S D=.61$ weeks), and it included 19 boys and 17 girls. The data from 16 additional 4-month-old infants were not used because they fussed $(n=10)$, because they were inattentive $(n=5)$, or because of equipment failure $(n=1)$. The mean age of the 6-month-old group was 28.4 weeks ( $S D=.74$ weeks), and it included 18 boys and 18 girls. The data from 12 additional 6-month-old infants were not used because they fussed or cried $(n=7)$, because they were inattentive $(n=3)$, or because the parent interfered with the infant during the test $(n=2)$. The mean age of the 8-month-old group was 37.2 weeks ( $S D=.58$ weeks), and it included 19 boys and 17 girls. The data from 8 additional 8-month-old infants were not used because of fussing $(n=7)$ or because the parent interfered with the infant $(n=1)$. The mean age of the 10-month-old group was 46.4 weeks ( $S D=.81$ weeks), and it included 18 boys and 18 girls. The data from 11 additional 10-month-old infants were not used because of fussing $(n=3)$, because of inattentiveness $(n=3)$, or because the parent interfered with the infant $(n=5)$. All the infants were healthy at the time of testing and were full-term at the time of birth, with birth weights greater than $2,500 \mathrm{~g}$, gestational ages greater than 37 weeks, and Apgar scores greater than 7 .

Apparatus and Stimuli. During testing, each infant sat in front of a 25-in. (measured diagonally) video monitor. The 4-month-old infants sat in an infant seat that was reclined at a $45^{\circ}$ angle with respect to the monitor. One 4-month-old infant refused to sit in the infant seat and, as a result, was tested on the parent's lap. The 6-, 8-, and 10-month-old infants either sat in a commercially available high chair or in a parent's lap. Seven of the 6-month-old, 11 of the 8-month-old, and 13 of the 10-month-old infants refused to sit in the high chair and, therefore, were tested on the parent's lap. For those infants who sat in the infant seat or the high chair, the parent was seated behind them and out of his/her sight. The parents who held their infants during the test were requested to sit as 
still as possible and to refrain from any interactions with their babies. If the parent did not comply with the request, the infant's data were discarded.

The monitor was enclosed on both sides with a curtain that extended out on each side past where the infant was sitting. The front of the video monitor was covered with a posterboard panel that had a rectangular window cut out of it that measured $7.5 \mathrm{~cm}$ in width and $34 \mathrm{~cm}$ in height. The infant was seated directly in front of the monitor and had to look $45^{\circ}$ to his/her left to view the visual stimulus. The distance from the infant to the stimulus was $50.5 \mathrm{~cm}$. The visual stimuli were computer-generated "sprite" graphics presented on the video monitor. They were produced by a Supersprite video display board running inside an Apple Ile microcomputer. During each trial, a single stimulus object could be seen moving up and down in the window. The stimulus was a circular twodimensional green image that subtended $3^{\circ} 48^{\prime}$ of visual angle. The distance traversed by the visual stimulus from top to bottom was $31.5 \mathrm{~cm}$. The auditory stimulus was generated by a sound-generating chip on the Supersprite board. It was a complex tone whose envelope descended in time. The overall duration of the auditory stimulus was $271 \mathrm{msec}$, and it measured $63 \mathrm{~dB}$ (re .0002 dynes $/ \mathrm{cm}^{2}$, A scale) at the infant's ear. Spectrum analysis indicated that the sound had a fundamental frequency of $62.5 \mathrm{~Hz}$ and several harmonic peaks.

The auditory stimulus was presented through the two built-in speakers located on each side of the monitor. A baffle, oriented at a $45^{\circ}$ angle with respect to the side of the monitor and located behind each speaker, projected the sound forward toward the infant. To attract the infant's attention to the window, a schematic face was displayed in the center of the window prior to the start of each trial. A video camera, located on top of the video monitor, provided the observer with a picture of the infant. Although all scoring was done on line, the entire test session was also videotaped.

Procedure. Each infant was tested individually in a dimly illuminated, quiet room. The ambient sound pressure level in the room, as measured at the infant's ear, was $56 \mathrm{~dB}$ (re .0002 dynes $/ \mathrm{cm}^{2}$, A scale). An infant-controlled habituation procedure was used. The testing session began as soon as the infant was seated. The moment the infant looked at the schematic face in the monitor window, the face disappeared and the visual stimulus appeared at the top of the screen and began to move in the downward direction. The off-axis distance of $50.5 \mathrm{~cm}$ between the infant and the stimulus on the screen was used to calculate the velocities of the visual stimuli. For half the infants, the visual stimulus moved at $23^{\circ} 55^{\prime}$ of visual angle/sec. For the other half, the stimulus moved at $54^{\circ} 10^{\prime}$ of visual angle/sec. At the same time that the visual stimulus reached the end of its downward trajectory, the auditory stimulus sounded and the stimulus reversed its direction and began to travel upward. There was no pause in the motion of the visual stimulus when the auditory stimulus sounded. For those infants who were habituated with the slow visual stimulus, the sound occurred at a rate of $.325 \mathrm{~Hz}$ (or every $3.08 \mathrm{sec}$ ), whereas for those infants habituated with the rapid stimulus, the sound occurred at a rate of $.73 \mathrm{~Hz}$ (or every $1.37 \mathrm{sec}$ ). Whichever rate was not used during habituation became the novel rate presented during the test trials.

As long as the infant looked at the window, the visual stimulus continued to move up and down and the auditory stimulus sounded each time the visual stimulus reached bottom. As soon as the infant looked away for more than $1 \mathrm{sec}$, the visual stimulus disappeared, the sound ceased, and the trial ended. Once the trial ended, the schematic face reappeared. The next trial began when the infant looked back at the face in the window. The total number of habituation trials was contingent on a predetermined decline in the duration of looking. Specifically, the total duration of looking during the last three trials had to decline to less than $50 \%$ of the total duration of looking during the first three habituation trials. As a result, a minimum of six habituation trials was administered to each infant. Once the criterion was met, the next trial initiated the test phase.

Three types of test trials were administered to each infant: auditory (A), where the repetition rate of the auditory component was changed to the novel rate while the rate of the visual component was not changed; visual (V), where the rate of motion of the visual stimulus was changed by changing its velocity of motion to the novel velocity but the rate of the auditory component was not changed; and auditory/visual (AV), where the rate of both components changed to the novel rate. As noted earlier, a change in the synchrony relation between the two components accompanied the change in the $A$ and $V$ test trials. Each type of test trial was administered twice in succession, with three refamiliarization trials presented between each set of the two test trials. During the refamiliarization trials, the compound stimulus originally presented during the habituation phase was presented again. The order of the three sets of test trials was counterbalanced across infants within each age group. As a result, each age group was divided into six subgroups, with each subgroup receiving one of six possible orders of the three types of test trials. As in the habituation phase, the length of each trial during the test phase was controlled by the infant.

The experimenter, who was located out of the infant's view, observed the infant on a second video monitor. While observing the infant's eyes, the experimenter controlled the presentation of the stimuli by initiating trials whenever the infant looked at the schematic face in the window. Because the number of habituation trials could vary in an unpredictable manner across infants, there was no way for the experimenter to know when the habituation phase was over and when the test phase began. Also, the experimenter could not see the stimulus because she could not see the monitor on which the stimulus was displayed, nor could she hear it because she wore headphones and listened to music throughout the test session.

\section{Results}

Because each infant received each type of test trial twice, a mean looking score, based on the total amount of looking in each of the two trials, was computed for each type of test trial. In addition, a mean looking score was computed for the last two habituation trials. Because inspection of these mean scores indicated that they were positively skewed, they were normalized through a log transform and all subsequent analyses were based on the transformed scores. The mean score computed for the last two habituation trials constituted a baseline measure of responsiveness against which responsiveness in each of the test trials was compared to determine if significant response recovery occurred.

There were two questions that the analyses were designed to answer: (1) Did the infants discriminate any of the changes introduced during the test trials? and (2) Were there any differences in the infants' response to the different types of changes? To answer the first question, the magnitude of response in the baseline trials and in each of the three test trials was entered into a four-way, repeated measures multivariate analysis of variance (MANOVA), with trial type as the within-subject factor and age, habituation group (slow or fast habituation stimulus), and test trial order as the between-subject factors. As can be seen in Figure 1, response magnitude differed significantly as a function of trial type [Wilks's $\delta=.248$, Rao $R(3,94)$ $=95.01, p<.001]$. There was also a significant trial 


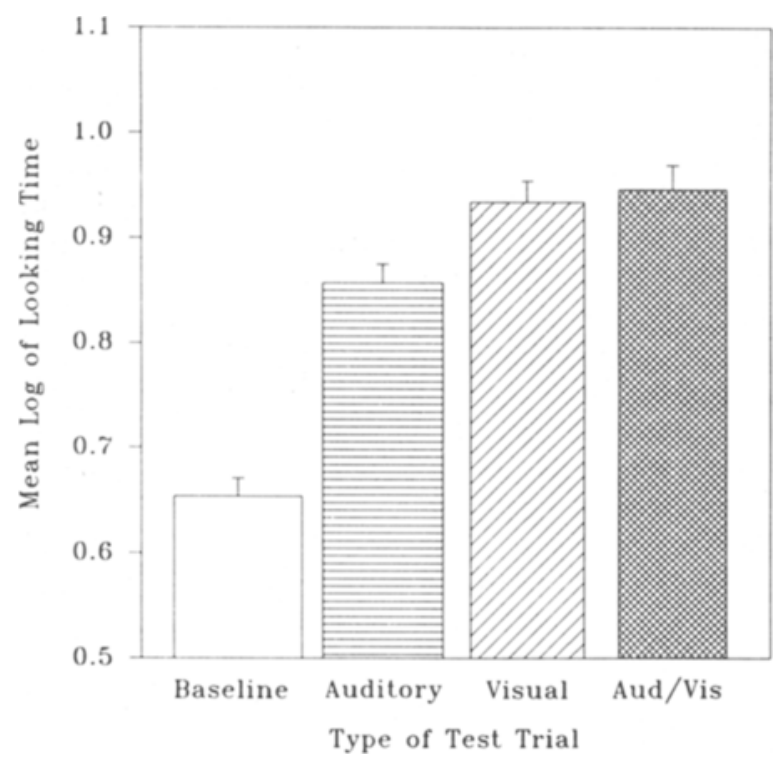

Figure 1. Mean log of looking time in the baseline trials and in each of the three test trials in Experiment 1. Error bars represent standard errors of the mean.

type $\times$ trial order interaction [Wilks's $\delta=.622$, Rao $R(15,259)=3.25, p<.001]$, indicating that the magnitude of response on a given type of test trial depended on the ordinal position of that particular test trial with respect to the other two types of test trials.

To explore the main effect of trial type, and to determine whether there was significant response recovery in each of the test trials, the response in each type of test trial was compared with the baseline response by way of the Tukey $L S D$ post hoc test. The post hoc comparisons showed that there was significant response recovery in each of the test trials (all ps $<.001$ ). Although these comparisons indicated that there was significant response recovery in each test trial, the trial order $\times$ trial type interaction raised the possibility that significant response recovery may have occurred for some ordinal positions but not for others. As a result, a covariance analysis was performed to determine if this was the case. This analysis indicated that response recovery remained statistically significant even when test trial order was covaried out as a factor. As in the original analysis, there was a significant main effect of trial type [Wilks's $\delta=.424$, Rao $R(3,227)=102.81, p<.001]$. The Tukey $L S D$ post hoc test indicated that response recovery was statistically reliable in the A, V, and AV test trials (all $p s<.001$ ). These results indicate that the infants exhibited significant discrimination in each of the test trials regardless of the ordinal position in which a given test trial was administered.

The foregoing pattern of results was reflected in individual infants' responses. Analyses comparing each infant's response in each of the three test trials to his/her response in the habituation trials indicated that the effects found in the group data were highly consistent across in- fants. Out of 144 infants, 120 increased their looking in the A test trial, 128 increased their looking in the V test trial, and 128 increased their looking in the AV test trial (binomial $p$ s $<.001$ ).

The second question, regarding possible differences in response in the different test trials, was answered by comparing the magnitude of response recovery attained in each test trial. Because a recovery score represents the difference between the baseline score and the score in each of the test trials, respectively, comparisons of recovery scores are the most appropriate way to answer the second question because they are made relative to a common anchor point. The recovery scores were submitted to a four-way, repeated measures MANOVA, with age, test trial order, and habituation group as the betweensubject factors and test trial type as the within-subject factor.

The results of this analysis indicated that there was a main effect of trial type [Wilks's $\delta=.80$, Rao $R(2,95)$ $=11.64, p<.001]$. The Tukey $L S D$ post hoc test indicated that the magnitude of response recovery was significantly greater in the $\mathrm{V}(p<.001)$ and $\mathrm{AV}(p<.001)$ test trials than in the $A$ test trial. In addition to the main effect of trial type, there was also a significant test trial type $\times$ test trial order interaction [Wilks's $\delta=.635$, Rao $R(10,190)=4.84, p<.001]$. A covariance analysis, with test trial order as the covariate, indicated that the main effect of trial type was still significant [Wilks's $\delta$ $=.87$, Rao $R(2,133)=10.15, p<.001$ ], and the Tukey $L S D$ post hoc test indicated again that the magnitude of response recovery in the $\mathrm{V}$ and $\mathrm{AV}$ test trials was significantly greater than in the A test trial $(p<.01$ for the $\mathrm{A}-\mathrm{V}$ contrast, and $p<.001$ for the $\mathrm{A}-\mathrm{AV}$ contrast).

Additional analyses were conducted to clarify the test order effect. The data from the three types of trials were inspected separately for each order to determine whether any systematic trends emerged. The most clear-cut effect that emerged was that the magnitude of responsiveness in the AV test trial was dependent on its ordinal position. That is, the infants responded most when the AV test trial was first in the sequence of test trials, less when it was preceded by a $V$ test trial, and least when it was preceded by an A test trial. A one-way ANOVA, with ordinal position of the AV test trial as the between-subject factor, confirmed that these differences were statistically reliable by indicating that ordinal position was a significant effect $[F(2,141)=6.76, p<.01]$. Post hoc contrasts indicated that the magnitude of response in the AV test trial when it occurred first was significantly greater than when it occurred after the $\mathrm{V}$ test trial $[F(1,141)=18.46, p<$ $.001]$ or when it occurred after the A test trial $[F(1,141)$ $=62.91, p<.001]$. Also, the magnitude of response in the AV test trial was greater when it occurred after the $V$ test trial than when it occurred after the A test trial $[F(1,141)=6.61, p<.05]$.

Although the main purpose of the refamiliarization trials was to give the infants an opportunity to become reacquainted with the original compound stimulus, the data 
from these trials also can be used to ask whether infants' responsiveness remained at habituation levels. A comparison of the magnitude of response in the last refamiliarization trial in each set of refamiliarization trials and the magnitude of response in the baseline trials indicated that there was no difference and, thus, that habituation was reestablished.

No age-specific analyses were conducted in any of the foregoing analyses because age was not a significant factor. Nonetheless, it is important to determine whether the significant response recovery observed in the three types of test trials was due to the pooling of the data from all the age groups or whether it actually occurred at each of the ages tested. Comparisons of the response in each test trial versus the response in the baseline trials revealed that there was, indeed, a significant response recovery in each of the three types of test trials $(p s<.01)$ in each age group. Examination of the individual infants' responses indicated that out of 36 infants at each age, the following number exhibited recovery in the A, V, and AV test trials, respectively: 36,35 , and 36 at 4 months, 28,31 , and 33 at 6 months, 28, 33, and 31 at 8 months, and 28, 29, and 28 at 10 months (binomial $p s<.001$ ).

\section{Discussion}

The results from this study indicate that infants as young as 4 months of age can discriminate a combined-modality change as well as a change in each of the component modalities when the unimodal changes occur against a background of no change in the second modality. The capacity to make these discriminations was present at each of the ages tested, and there were no age differences in the degree to which the infants responded to the different types of changes. Although no age differences were found, differences in responsiveness to the different types of changes were found for the group as a whole. The infants responded less to the auditory change than they did to the visual or to the combined change, and they responded equivalently to the visual and to the combined changes. This suggests that the type of change that the infants experienced in the visual modality in the current study was more salient than was the type of change that they experienced in the auditory modality. The lack of a difference in magnitude of response between the $V$ and $A V$ test trials suggests that the greater response in the AV test trial relative to the A test trial was due to the change in the visual component.

An interesting and rather surprising finding was that the ordinal position of a given test trial affected the magnitude of responsiveness. This effect did not, however, prevent the infants from being able to discriminate the various changes, because significant discrimination was found in all three test trials even when test trial order was controlled statistically.

\section{EXPERIMENT 2}

The advantage of the infant-controlled habituation procedure is that it ensures that all infants reach the same degree of familiarity with the habituation stimulus. The one drawback of this procedure, however, is that it introduces the possibility that the observed recovery of response during a novel test trial may, in part, be due to regression to the mean. The fact that the magnitude of response during the last refamiliarization trials in Experiment 1 did not differ from habituation levels suggests that regression to the mean did not play a role. Nonetheless, because the refamiliarization trials are not a direct way of answering the question of regression to the mean, it was felt that an independent investigation of this question was warranted, with a design that was a more direct test of it.

As a result, a second experiment was carried out in which infants were tested in the identical manner except that, once an infant met the habituation criterion, two additional habituation trials were administered. If regression to the mean were operating, response magnitude should increase in these two "lag" trials compared with the response magnitude observed in the two directly preceding habituation trials. Conversely, if regression to the mean were not operating, responding should remain at the habituation criterion level. Because inclusion of the test trials following the lag trials imposed no additional "cost," these were included in this experiment so as to provide an opportunity to determine the reliability of the findings obtained in the first experiment.

\section{Method}

Subjects. Groups of 24 infants each were tested at 4, 6, and 8 months of age. The mean age of the 4-month-old group was 19.9 weeks ( $S D=.68$ weeks), and it consisted of 13 boys and 11 girls. The data from 14 additional 4-month-old infants were not used because they fussed $(n=10)$, because they were inattentive $(n=$ $1)$, or because they were sleepy $(n=3)$. The mean age of the 6month-old group was 28.1 weeks ( $S D=.8$ weeks), and it consisted of 13 boys and 11 girls. The data from 5 additional 6-monthold infants were not used because they fussed or cried $(n=4)$ or because they were inattentive $(n=1)$. The mean age of the 8-monthold group was 36.9 weeks ( $S D=.83$ weeks), and it consisted of 9 boys and 15 girls. The data from 1 additional 8-month-old infant were not used because of distractions.

Apparatus and Stimuli. The apparatus and stimuli used in this experiment were identical to those used in Experiment 1.

Procedure. The procedure used in this experiment was identical to the procedure used in Experiment 1 with one exception. Once each infant met the habituation criterion specified in Experiment 1 (a 50\% decrease in looking duration during the last three trials compared with the first three trials), two additional habituation trials (lag trials) were administered. Following completion of the two lag trials, the identical series of test trials that was administered in Experiment 1 also was administered in this experiment.

\section{Results}

The first analysis compared the magnitude of response during the lag habituation trials with the magnitude of response during the two directly preceding trials, which in the previous study were designated as the baseline trials. This was done by means of a four-way, repeated measures MANOVA, with habituation trial type as the withinsubject factor and age, test trial order, and habituation 


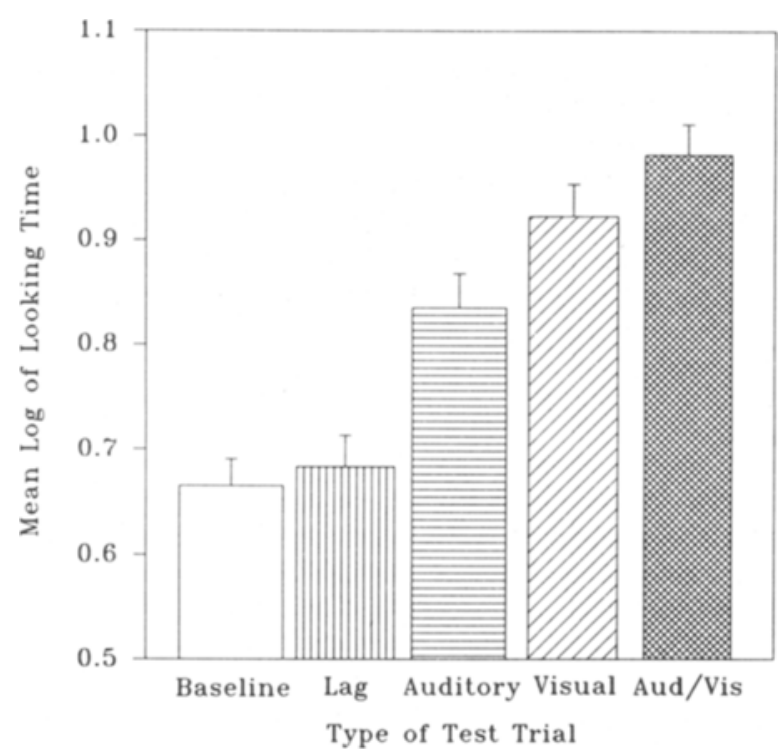

Figure 2. Mean log of looking time in the lag habituation trials, baseline trials, and each of the three test trials in Experiment 2. Error bars represent standard errors of the mean.

group as the between-subject factors. There was no significant main effect of habituation trial type $(p=.57)$, nor did trial type interact with any of the factors. Figure 2 shows the log-transformed data from these two types of trials. In terms of the raw looking time, the overall mean looking time was $5.2 \mathrm{sec}$ for the baseline trials and $5.9 \mathrm{sec}$ for the lag habituation trials. It is thus clear that spontaneous recovery of responsiveness did not occur in the present study.

The response in each of the test trials also can be seen in Figure 2. To determine whether the infants discriminated the changes that occurred during the test trials, response magnitude in each test trial and response magnitude in the lag habituation trials were all submitted to a four-way MANOVA, with trial type as the within-subject factor and age, habituation group, and test trial order as the between-subject factors. The MANOVA indicated that there was a significant main effect of trial type [Wilks's $\delta=.31$, Rao $R(3,34)=25.28, p<.001]$ and a marginally significant trial type $\times$ trial order interaction [Wilks's $\delta=.52$, Rao $R(15,94)=1.65, p=.074$ ]. The Tukey $L S D$ post hoc tests, comparing response magnitude in each of the test trials with that in the lag habituation trials, indicated that there was a significant recovery of response in the $A, V$, and AV test trials (all $p s<.001$ ). Although response in the different test trials was only marginally affected by the ordinal position of the test trials, a covariance analysis, with test trial order as the covariate, was still performed. Results of this analysis were identical to the results from the preceding analysis. There was a significant main effect of trial type [Wilks's $\delta=.456$, Rao $R(3,61)=24.21, p<.001]$, and there was significant response recovery in the $\mathrm{A}, \mathrm{V}$, and $\mathrm{AV}$ test trials (all $p s<.001$ ).
As in Experiment 1, the foregoing pattern of results was reflected in individual infants' responses. Analyses comparing each infant's response in each of the three test trials to his/her response in the lag habituation trials indicated that the effects found in the group data were highly consistent across infants. Out of 72 infants, 53 increased their looking in the A test trial, 56 increased their looking in the $\mathrm{V}$ test trial, and 61 increased their looking in the AV test trial (binomial $p s<.001$ ). Out of 24 infants at each age, the following number exhibited recovery in the $A$, $\mathrm{V}$, and AV test trials, respectively: 16,19 , and 19 at 4 months, 20,18, and 22 at 6 months, and 17, 19, and 20 at 8 months (all binomial $p$ s $<.05$, except in the A test trial in the 4-month-olds, where $p<.10$ ).

To determine whether response magnitude differed in the different test trials, recovery scores were computed by taking the difference between response magnitude in each type of test trial and response magnitude in the lag habituation trials, respectively. The overall results of the four-way (trial type $\times$ age $\times$ habituation group $\times$ test trial order) MANOVA were highly similar to those obtained in Experiment 1. There was a significant main effect of trial type [Wilks's $\delta=.648$, Rao $R(2,35)=9.49$, $p<.001$ ], and Tukey $L S D$ post hoc tests indicated that response magnitude in the $\mathrm{V}$ and $\mathrm{AV}$ test trials was significantly greater than in the A test trial $(p<.05$ and $p<.001$, respectively). There was also a significant test trial type $\times$ test trial order interaction [Wilks's $\delta=.55$, Rao $R(10,70)=2.40, p<.025$ ]. A covariance analysis, with test trial order as the covariate, indicated that there was a significant main effect of trial type [Wilks's $\delta=.77$, Rao $R(2,63)=9.26, p<.001]$, and Tukey $L S D$ post hoc comparisons indicated that the response in the $\mathrm{V}$ and $\mathrm{AV}$ test trials was significantly greater than in the A test trial $(p<.05$ and $p<.001$, respectively).

As in Experiment 1, the data were inspected to clarify the effect of test trial order. Because the major effect identified in Experiment 1 was the effect of test trial order on responsiveness in the AV test trial, the data from this experiment were inspected to determine whether the same was true here. In contrast to Experiment 1, there was no main effect of trial order on the magnitude of response in the AV test trial. The magnitude of response in the AV test trial was greater, however, when it was first in the sequence than when it was preceded by the $\mathrm{V}$ test trial $[F(1,69)=11.91, p<.01]$ or the A test trial $[F(1,69)$ $=23.79, p<.001]$.

\section{Discussion}

The principal finding in the present experiment was that spontaneous recovery of responding due to regression to the mean did not account for the recovery of responsiveness observed during the test trials. The level of responsiveness reached during the last two trials of the habituation phase remained the same when two additional habituation trials were administered. Moreover, the findings from this study replicated the findings from Experiment 1 in virtually every respect. Not only were the main effects and significant response recovery in each type of 
test trial replicated, but even the test trial $\times$ test trial order interaction and the greater response to the visual component compared with the auditory component change were replicated. The replication of all these effects is all the more impressive given that two additional habituation trials were administered and given that the sample size in the current study was half the sample size in Experiment 1 .

\section{EXPERIMENT 3}

Although the $\mathrm{A}$ and $\mathrm{V}$ test trials are useful in assessing the contribution that each of the components makes to responsiveness, the results from these two test trials cannot be interpreted unambiguously. This is because whenever the rate of either component was changed, the sound no longer occurred in synchrony with the bounce of the object. In other words, the temporal relationship of the two components also changed. As a result, we conducted an experiment to determine whether the infants were sensitive to the change in the temporal relationship of the components. The same procedures were used, but this time the AV test trial was replaced by a new test trial where only the temporal relationship of the two components was changed. Thus, infants were habituated to a compound stimulus whose components were synchronous, and then they were given the two unimodal test trials where the rate of the respective component changed and a third test trial during which the synchrony between the bounce of the visual stimulus and the occurrence of the auditory stimulus was disrupted.

\section{Method}

Subjects. Separate groups of 24 infants were tested at 4, 6, 8, and 10 months of age. The mean age of the 4-month-old group was 20.3 weeks ( $S D=5.6$ weeks), and it consisted of 14 boys and 10 girls. Data from 11 additional 4-month-old infants were not used because they fussed $(n=10)$ or were inattentive $(n=1)$. The mean age of the 6-month-old group was 29.7 weeks ( $S D=2.8$ weeks), and it consisted of 11 boys and 13 girls. Data from 4 additional 6-month-old infants were not used because they fussed $(n=3)$ or because they were sleepy $(n=1)$. The mean age of the 8-monthold group was 36.2 weeks ( $S D=3.1$ weeks), and it consisted of 13 boys and 11 girls. Data from 5 additional 8-month-old infants were not used because they were fussy $(n=2)$, because they were inattentive $(n=2)$, or because they were distracted $(n=1)$. The mean age of the 10-month-old group was 46.4 weeks ( $S D=5$ weeks), and it consisted of 12 boys and 12 girls.

Apparatus and Stimuli. The apparatus and stimuli were identical to those used in Experiment 1.

Procedure. The procedure was identical to the procedure used in Experiment 1 except that the AV test trial was replaced by an $\mathrm{AV}_{\text {(async) }}$ test trial where only the temporal relationship of the two components was changed. As a result, for the group habituated with the slow compound stimulus, the sound occurred $850 \mathrm{msec}$ after the visual stimulus began to move down from its starting position at the top. For the group habituated with the fast compound stimulus, the sound occurred $350 \mathrm{msec}$ after the visual stimulus began to move down. In both cases, the occurrence of the sound was timed to occur at the halfway point in the downward motion of the visual stimulus.

\section{Results}

Analysis of test trial scores indicated that there was an overall effect of trial type [Wilks's $\delta=.43$, Rao $R(3,46)$ $=20.59, p<.001$ ] (see Figure 3 ). In contrast to the other two studies, there was no significant test trial $x$ trial order effect. Tukey $L S D$ post hoc tests indicated that there was significant recovery of response in the $A, V$, and $A V_{\text {(async) }}$ test trials (all $p s<.001$ ). Comparison of the recovery scores from each of the test trials indicated that there was a significant effect of trial type [Wilks's $\delta$ $=.74, \operatorname{Rao} R(2,47)=8.37, p<.001]$, and Tukey $L S D$ post hoc tests indicated that the difference between the $A$ and $V$ test trials approached significance $(p<.07)$ and that there was a significant difference between the $\mathrm{V}$ and $\mathrm{AV}_{\text {(async) }}$ trials $(p<.001)$.

Analysis of individual subjects' responses showed that out of 96 infants, 74 looked longer in the A test trial, 81 looked longer in the $\mathrm{V}$ test trial, and 69 looked longer in the $A V_{\text {(async) }}$ test trial. Out of 24 infants at each age, the following number exhibited recovery in the $A, V$, and $A V_{\text {(async) }}$ test trials, respectively: 19,18 , and 21 at 4 months, 18, 20, and 16 at 6 months, 20,21, and 14 at 8 months, and 17,22 , and 18 at 10 months.

The significantly smaller response in the $A V_{(\text {async) }}$ test trial relative to the $\mathrm{V}$ test trial is different from the response obtained in the other two experiments where the combined change yielded a response equivalent to that obtained in the V test trial. To determine directly whether the response pattern was different in the two experiments, the recovery scores from Experiment 1 and the recovery scores from the current experiment were compared by way of a repeated measures MANOVA, with trial type

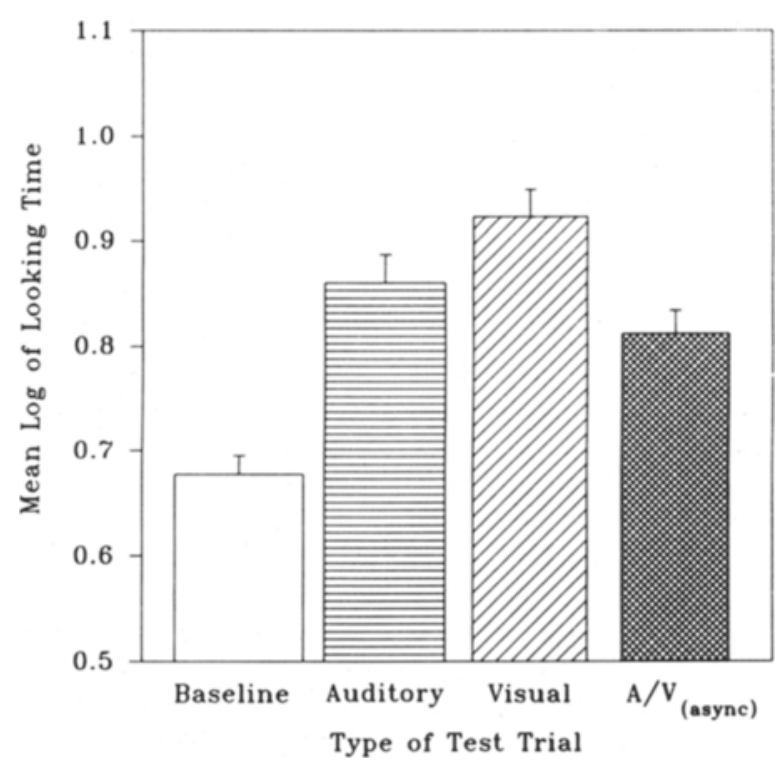

Figure 3. Mean log of looking time in the baseline trials and in each of the three test trials in Experiment 3. Error bars represent standard errors of the mean. 
as the within-subject factor and age, habituation group, test trial order, and experiment as the between-subject factors. The results of this analysis indicated that there was a significant test trial $\times$ experiment interaction [Wilks's $\delta=.87$, Rao $R=10.79, p<.001$ ], and the Tukey $L S D$ post hoc test indicated that the source of the interaction was a significantly greater response in the AV test trial than in the $\operatorname{AV}_{\text {(async) }}$ test trial $(p<.001)$.

\section{Discussion}

The results from the unimodal test trials in this study replicate the results from the previous two studies in showing that infants between 4 and 10 months of age can discriminate changes in the rate of a moving visual stimulus against an unchanging auditory background and can discriminate changes in the rate of a periodically occurring sound against a background of an unchanging moving visual stimulus. The results from the $\mathrm{AV}_{\text {(async) }}$ test trial indicated that the change in the temporal relationship between the auditory and visual components was discriminable, but a comparison with the results from the AV test trial indicated that the change in the rate of both components elicited a greater response than did a change in the synchrony relation.

\section{GENERAL DISCUSSION}

A fairly common perceptual event in an infant's life is a moving object that produces a sound when it changes its trajectory as it encounters an obstacle. The present experiments were designed to mimic such an event in an effort to find out how infants respond to the different aspects of this multimodal event. The basic finding in all three experiments was that at each of the ages tested, infants between 4 and 10 months of age discriminated a change in the temporal properties of each component as well as a simultaneous change in the temporal properties of both components.

Although the ordinal position of the test trials in Experiments 1 and 2 affected responsiveness to the changes in the test trials, it affected only the magnitude of responsiveness, not the ability to perform the discrimination. Because the ordinal position of each type of test trial was completely counterbalanced across infants, this effect could not have contributed to the differential responsiveness to the different types of changes. Moreover, the same pattern of responsiveness was obtained when test trial order was controlled statistically. Post hoc examination of responsiveness in each type of test trial as a function of its ordinal position revealed that in both Experiments 1 and 2, responsiveness in the AV test trial was lower when the AV test trial was preceded by either of the unimodal changes than when the AV test trial was first. The fact that a similar effect was not found in Experiment 3 suggests that this effect is due to the specific nature of the combined-modality change; a simultaneous change in the rate of both components appears to be more effective than a simultaneous change in the temporal relationship of the two components.

The fact that experience with a change in one attribute of the compound stimulus had an effect on subsequent responding to a change in another attribute is interesting, because the effect occurred despite the interposition of three refamiliarization trials between the test trials. This suggests that the infants had to remember a change in a multimodal compound stimulus across a period of time when they were exposed to what might be considered interfering stimuli. In other words, it seems that a greater capacity to remember would be required in this case than that needed to make a discriminative response in the common form of the habituation/test procedure where the novel stimulus directly follows the familiar stimulus.

When the data from all three experiments are considered together, two conclusions emerge: (1) changes in rate wrought by spatial variations of the visual component were more salient ${ }^{1}$ than were changes in rate wrought by temporal variations in the repetition rate of the sound, and (2) the temporal property of rate influenced responsiveness to a greater degree than did the temporal property of synchrony. These conclusions are warranted for the following reasons.

The finding that response in the $V$ test trial was greater than in the A test trial suggests that the change in the visual component was more salient. It should be remembered, however, that this is a relative difference in response recovery rather than a difference derived from a direct test that pits the visual change against the auditory change. Although it is legitimate to conclude that a change in the visual component was more salient than a change in the auditory component, it is not clear whether it was rate or synchrony that was responsible for response recovery, because synchrony and rate changed simultaneously in each unimodal test trial. One way to disentangle the role of synchrony and rate is to examine the results from the $A V_{\text {(async) }}$ test trial. These results show that synchrony played a much more limited role in responsiveness than did rate for two reasons. One reason was that a bimodal rate change without a concomitant synchrony change (AV test trial) resulted in a greater response than did a synchrony change without a concomitant rate change (the $\mathrm{AV}_{\text {(async) }}$ test trial). The second reason was that a concomitant synchrony and rate change (the $\mathrm{V}$ test trial) elicited a greater response than did a synchrony change alone (the $A V_{\text {(async) }}$ test trial). The fact that the combined synchrony and rate change in the A test trial did not elicit a greater response than the synchrony change in the $A V_{\text {(async) }}$ test trial also suggests, like the $A$ and $V$ comparison did, that the rate change in the auditory component was not as salient as the rate change in the visual component.

To determine further whether the change in the visual component was more salient than the change in the auditory component, responding in each unimodal trial, respectively, can be compared with responding in the bi- 
modal test trial. This comparison asks whether a change in synchrony combined with a change in the rate of one component was as salient as a bimodal change in rate. The data showed that the change in the visual component and its temporal relationship to the auditory component was as salient as the bimodal rate change, but that a change in the auditory component and its temporal relationship to the visual component was less salient than the bimodal rate change. This difference once again suggests that the change in the visual component was more salient. It also suggests that the increased recovery in the AV test trial relative to the $A$ test trial was primarily due to the change in the rate of the visual component. This conclusion is also consistent with the finding that the response magnitude in the $A V_{\text {(async) }}$ test trial, where no rate change $O c-$ curred, was significantly lower than in the $V$ test trial and was equal to that obtained in the A test trial.

It might be argued that the greater responsiveness to the visual as opposed to the auditory component reflects an inherent bias of the methodology employed in the current experiments. Because this methodology relies on the infants' visual responses, it might be argued that this might bias responsiveness toward changes in the visual modality. This bias, however, is unlikely because the same methodology yielded greater responsiveness to changes in the auditory modality in earlier studies (Lewkowicz, 1988a, 1988b).

On the basis of what has come to be known as the "redundant-signals effect" in adults (Miller, 1982; Raab, 1962), it might be expected that the bimodal change would result in greater responding. The redundant-signals effect is characterized by faster responding to bimodal than to unimodal signals such that bimodal inputs appear to confer an advantage over unimodal inputs in localization tasks (Stein, Meredith, Huneycutt, \& McDade, 1989). A comparison of response magnitude in each type of test trial did not, however, provide evidence of a redundant-signals effect even though both unimodal changes were discriminable. The data indicated that the response in the AV test trial in Experiments 1 and 2 was statistically greater than the response in the A test trial, but that it was not greater than the response in the V test trial. Given that the auditory component appeared to be less salient than the visual one, it is likely that the redundant-signals effect would probably have been obtained had the auditory component been as salient as the visual one. The absence of the redundant-signals effect was not likely to be due to a "ceiling effect" in the AV trial because the infantcontrolled method is open ended in terms of duration of looking.

A comparison of the findings from the current experiments and those from Lewkowicz's (1988a, 1988b) experiments reveals some interesting differences that are consistent with the modality appropriateness hypothesis. Although the 6-month-old infants in the earlier experiments did not respond to temporal changes in the visual component, infants as young as 4 months of age did respond to the temporal changes in the current experiments.
In fact, the response in the current experiments was robust, and the finding was replicated across three different experiments. The one key difference between the earlier experiments and the current ones is that the visual component moved in the current experiments but was spatially static in the earlier ones. As noted earlier, the visual modality is highly responsive to spatial variations, and, as a result, the finding of successful discrimination of changes in the rate of motion was not surprising. In fact, sensitivity to motion appears to be present at birth (Kaufmann, Stucki, \& Kaufmann-Hayoz, 1985; Kremenitzer, Vaughan, Kurtzberg, \& Dowling, 1979; Volkmann \& Dobson, 1976). Moreover, motion appears to be a fundamental property of the visual world, and motion-detection mechanisms are found at many phylogenetic levels where pattern-detection mechanisms are not available at all, or are very rudimentary (Nakayama, 1985). Similarly, in human development, motion- and temporal-detection mechanisms become differentiated earlier and are more adult-like earlier than are spatial-detection mechanisms (Banks \& Salapatek, 1983; Freedland \& Dannemiller, 1987). Thus, it would be expected that detection of changes in the visual component would be relatively easy.

Because the relative salience of the components was not matched either in the current experiments or in Lewkowicz's (1988a, 1988b) experiments, it is not yet possible to fulfill the goal of determining the relative standing of the sensory modalities in early development (Lewkowicz, 1991). Determination of sensory hierarchies in early development will have to await studies where the heteromodal components are matched in terms of their salience a priori or studies where heteromodal inputs that elicit conflicting responses are put in direct competition with one another. What the results from Lewkowicz's (1988a, 1988b) experiments and from the current ones show, however, is that the specific nature of the components making up a compound auditory/visual stimulus plays a major role in infants' responsiveness and that the nature of their response to different types of heteromodal inputs can be predicted on the basis of our knowledge about sensory system specialization.

\section{REFERENCES}

Allen, T. W., WAlker, K., Symonds, L., \& MARCell, M. (1977). Intrasensory and intersensory perception of temporal sequences during infancy. Developmental Psychology, 13, 225-229.

AsLin, R. N., SheA, S. L. (1990). Velocity thresholds in human infants: Implications for the perception of motion. Developmental Psychology, 26, 589-598.

BAHRICK, L. E. (1987). Infants' intermodal perception of two levels of temporal structure in natural events. Infant Behavior \& Development, 10, 387-416.

BANKS, M. S., \& SAlapatek, P. (1983). Infant visual perception. In M. M. Haith \& J. J. Campos (Eds.), Handbook of child psychology (pp. 453-571). New York: Wiley.

Dannemiller, J. L., \& Freedland, R. L. (1991). Detection of relative motion by human infants. Developmental Psychology, 27, 67-78.

Freedland, R. L., \& Dannemiller, J. L. (1987). Detection of stimulus motion in 5-month-old infants. Journal of Experimental Psychology: Human Perception \& Performance, 13, 566-576. 
Freides, D. (1974). Human information processing and sensory modality: Cross-modal functions, information complexity, memory, and deficit. Psychological Bulletin, 81, 284-310.

Kaufmann, F., Stucki, M., \&aufmann-Hayoz, R. (1985). Development of infants' sensitivity for slow and rapid motions. Infant Behavior \& Development, 8, 89-98.

Kremenitzer, J., Vaughan, H., Kurtzberg, D. Dowling, K. (1979). Smooth-pursuit eye movements in the newborn infant. Child Development, 50, $442-448$.

Kubovy, M. (1988). Should we resist the seductiveness of the space:time::vision:auditory analogy? Journal of Experimental Psychology: Human Perception \& Performance, 14, 318-320.

Kuhl, P. K., \& Meltzoff, A. N. (1982). The bimodal perception of speech in infancy. Science, 218, 1138-1140.

Lederman, S. J., Thorne, G., \& Jones, B. (1986). Perception of texture by vision and touch: Multidimensionality and intersensory integration. Journal of Experimental Psychology: Human Perception \& Performance, 12, 169-180.

LEwKowicz, D. J. (1986). Developmental changes in infants' bisensory response to synchronous durations. Infant Behavior \& Development, 9, 335-353.

LEwkowicz, D. J. (1988a). Sensory dominance in infants: 1. Six-monthold infants' response to auditory-visual compounds. Developmental Psychology, 24, 155-171.

LEwKowicz, D. J. (1988b). Sensory dominance in infants: 2. Tenmonth-old infants' response to auditory-visual compounds. Developmental Psychology, 24, 172-182.

LEWKowICZ, D. J. (1991). Development of intersensory functions in human infancy: Auditory/visual interactions. In M. J. Weiss \& P. R. Zelazo (Eds.), Newborn attention: Biological constraints and the influence of experience (pp. 308-338). Norwood, $\mathrm{NJ}$ : Ablex.

LEwKowicz, D. J. (1992). Infants' response to temporally based intersensory equivalence: The effect of synchronous sounds on visual preferences for moving stimuli. Infant Behavior \& Development, 15, 297-323.

Lewkowicz, D. J., \& Turkewitz, G. (1980). Cross-modal equivalence in early infancy: Auditory-visual intensity matching. Developmental Psychology, 16, 597-607.

MArKs, L. E. (1978). The unity of the senses. New York: Academic Press.
Mendelson, M. J., \& Ferland, M. B. (1982). Auditory-visual transfer in four-month-old infants. Child Development, 50, 747-751.

Miller, J. O. (1982). Divided attention: Evidence for coactivation with redundant signals. Cognitive Psychology, 14, 247-279.

Nakayama, K. (1985). Biological image motion processing: A review. Vision Research, 25, 625-660.

O'Connor, N., \& Hermelin, B. (1972). Seeing and hearing and space and time. Perception \& Psychophysics, 11, 46-48.

RAAB, D. (1962). Statistical facilitation of simple reaction times. Transactions of the New York Academy of Sciences, 24, 574-590.

SPELKE, E. S. (1979). Perceiving bimodally specified events in infancy. Developmental Psychology, 15, 626-636.

Stein, B. E., Meredith, M. A., Huneycutt, W. S., \& McDade, L. (1989). Behavioral indices of multisensory integration: Orientation to visual cues is affected by auditory stimuli. Joumal of Cognitive Neuroscience, 1, 12-24.

Volkmann, F. C., \& Dobson, M. V. (1976). Infant responses of ocular fixation to moving visual stimuli. Journal of Experimental Child Psychology, 22, 86-99.

WELCH, R. B., \& WARREN, D. H. (1986). Intersensory interactions. In K. R. Boff, L. Kaufman, \& J. P. Thomas (Eds.), Handbook of perception and human performance: Vol. I. Sensory processes and perception (pp. 1-36). New York: Wiley.

WiLcox, B. M., \& CLAYTON, F. L. (1968). Infant visual fixation on motion pictures of the human face. Journal of Experimental Child Psychology, 6, 22-32.

\section{NOTE}

1. The use of this term is not meant to imply that the reason for the differential response recovery is known. It is not possible to determine the reasons for the differential response recovery without corroborating evidence because different degrees of responsiveness could be due to greater discriminability of the different changes or to one change being more interesting than the other.

(Manuscript received December 19, 1991; revision accepted for publication May 13, 1992.) 